\title{
Status report of the CMS Superconducting Coil Project
}

\author{
D. Campi, P. Fabbricatore, A. Hervé, I.L. Horvath, F. Kircher
}

\begin{abstract}
The CMS Superconducting Coil is designed for one of the two large experiments of LHC at CERN. This Coil, $12.5 \mathrm{~m}$ long, $6 \mathrm{~m}$ diameter and $2.7 \mathrm{GJ}$ stored energy is a common project of the CMS Collaboration [1]. It is a four-layer coil, equipped with a self-supporting conductor capable of carrying $20 \mathrm{kA}$ to reach the maximum potential field of $4 \mathrm{~T}$. It has been designed with a considerable contribution from CEASaclay for the engineering, ETH-Zuirich for the conductor, INFN-Genova for the winding and CERN for the general coordination and construction of the ancillaries. The project entered the construction phase one year ago. The civil engineering is well advanced and ready to accept part of the yoke components already built. The coil itself has finished the pre-industrialization phase and the construction is beginning in industry [2]. Most of the important contracts have been awarded and the foreseen schedule is now based on contractual engagements. A quick overview of the features of the project as well as a status report of the main activities will be given.
\end{abstract}

Index Terms - Detector Magnets, Coils, LHC Project, Al stabilized conductor.

\section{INTRODUCTION}

CMS, Compact Muon Solenoid, is a general purpose detector designed to run at the highest luminosity $\left(\mathrm{L}>10^{34} \mathrm{~cm}^{-2} \mathrm{~s}^{-1}\right)$ at the LHC. The performance of the detector relies on the magnetic field generated by the Solenoidal Superconducting Magnet. The operating field requested by the CMS Collaboration is between 3.5 to $4 \mathrm{~T} ; 3.5 \mathrm{~T}$ being the minimum field required for good physics. The design of the Magnet has been carried out to maximize the chance of reaching $4 \mathrm{~T}$. The magnetic flux is generated by a 4-layer Superconducting Coil and returned by a $1.5 \mathrm{~m}$ thick iron Yoke which acts also as the supporting structure of the different CMS sub-detectors.

The CMS Magnet Project is a common project of the Institutes participating to the CMS Collaboration and is technically divided into two large parts: the Yoke Project and the Coil Project. The Superconducting Coil is based on the conceptual design of CEA-Saclay [3], with heavy participation of other Institutes which are technically and financially involved in producing the detailed design of the Coil and assure the industrial follow up of the major contracts. The CERN team is in charge of the general coordination and of the design of the Coil Ancillaries, like

Manuscript received September 19, 2000.

D. Campi and A. Hervé are with CERN, 1211 Geneva - Switzerland (telephone: +41-22-767.6607, e-mail: domenico.campi@cern.ch).

P. Fabbricatore is with INFN-Genova.

I.L. Horvath is with ETH-Zürich.

F. Kircher is with CEA-Saclay. the external cryogenics, the powering circuit and the controls, in order to optimize the operational aspects in the frame of the whole LHC Project.

The major contracts for the CMS Coil have been awarded by ETH-Zürich, FERMILAB-Batavia, INFN-Genova and CERN-Geneva. The Coil Project, formally approved in 1995, has just finished most of the pre-industrialization activities. This phase has permitted to check and validate most of the challenging technologies that will be used for the construction of the Coil, in particular those related with the conductor fabrication and the winding operation. The adjudication of contracts is also well advanced. More than $70 \%$ of the coil value is contractually engaged and within estimates.

The construction of the CMS Coil is foreseen to finish in late 2003 to be ready for the Magnet test during the first half of 2004 in the surface building of the CMS Experimental complex.

The main geometrical and operational parameters of the CMS Superconducting Coil are reported in Table I.

\section{CONDUCTOR}

One distinctive feature of the CMS coil design is the use of a reinforced conductor [5] which permits to react the magnetic and thermal efforts locally, without transmitting them through the whole winding pack and in particular through the insulation. The conductor is thus made from a 32-strand Rutherford type cable embedded in a $4 \mathrm{~N} 8$ pure $\mathrm{Al}$ stabilizer forming together the so-called insert. The insert is reinforced, through continuous electron beam welding, by two $6082 \mathrm{AA}$ sections. The conductor layout is shown in Fig. 1. The general development and construction of the conductor are mainly assured by ETHZ with the participation of Fermilab and CERN.

TABLE I

COIL PARAMETERS

\begin{tabular}{ll}
\hline & \\
Magnetic length & $12.5 \mathrm{~m}$ \\
Internal diameter & $6.3 \mathrm{~m}$ \\
Winding thickness & $262 \mathrm{~mm}$ \\
Magnetic induction at center & $4 \mathrm{~T}$ \\
Peak induction on the conductor & $4.6 \mathrm{~T}$ \\
Nominal current & $19.5 \mathrm{kA}$ \\
Magnetomotive force & $42.5 \mathrm{MAt}$ \\
Stored energy & $2.67 \mathrm{GJ}$ \\
Self-inductance & $14.05 \mathrm{H}$ \\
Weight of the cold mass & $225 \mathrm{t}$ \\
\hline
\end{tabular}




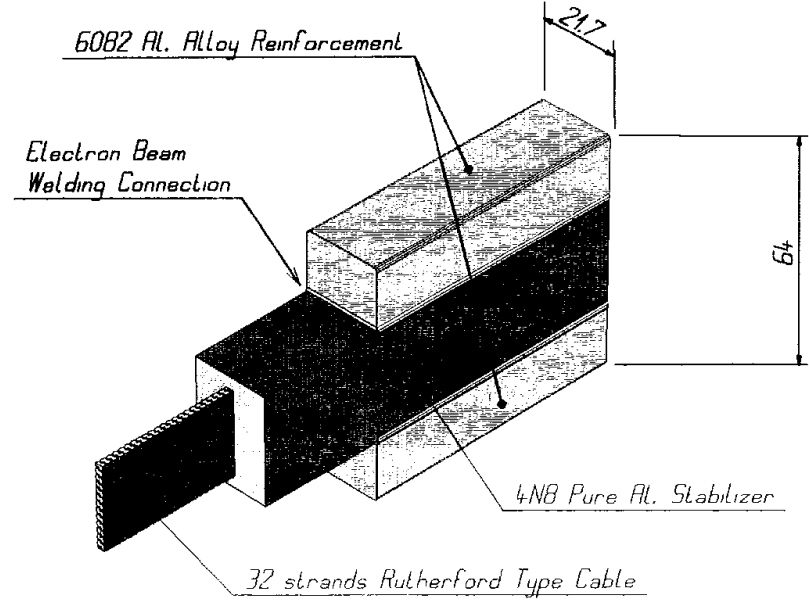

Fig. 1. AA reinforced Superconducting Conductor for the CMS Coil.

The purchasing and construction of the CMS conductor, are organized in the following way:

- The superconducting strands have been ordered to Outokumpu (Finland), for $60 \%$ of the total need, and to IGC Advanced Technologies (USA) for $40 \%$. The contracts have been awarded by Fermilab and first deliveries occurred in the past months satisfying the requested Ic of $1925 \mathrm{~A}$ at $5 \mathrm{~T}$, $4.2 \mathrm{~K}$, corresponding to a current density of $3140 \mathrm{~A} / \mathrm{mm}^{2}$ [1][5].

- The cabling of the strands has been ordered to Kabelwerke Brugg (Switzerland) and a frame contract has been established by ETHZ to perform the cabling of the $20+1$ spare unit lengths of Rutherford type cable. Each length is $2650 \mathrm{~m}$ long. First cabling trials have already been successfully performed and the company is now ready to undertake mass production.

- The 4N8 Pure Aluminum supply has been attributed, through a Fermilab contract, to Sumitomo (Japan) and a pure aluminum $(5 \mathrm{~N})$ has been delivered with an RRR of over 3000 at $0 \mathrm{~T}$ and around 1500 at $5 \mathrm{~T}$. Most of the Pure Aluminum has already been produced and delivered into billets ready for the co-extrusion of the insert.

- The co-extrusion of the so-called insert (Rutherford cable imbedded in high purity $\mathrm{Al}$ ) has been attributed, as a blanket contract, by ETHZ to Alcatel Cable Swiss at Cortaillod. This company, equipped with one of the few presses which permit a real continuous extrusion, has obtained very good results in terms of reliability. Geometrical tolerances are in the order of $0.1 \mathrm{~mm}$ and parallelism within $0.2 \mathrm{~mm}$. Two dummy sections of $2650 \mathrm{~m}$ have been produced, also in view of commissioning and testing the plant for the continuous electron-beam welding of the Aluminum Alloy reinforcement.

- The production of the 6082 Aluminum Alloy sections has been awarded to Alusuisse, Sierre (Switzerland) by Fermilab. An intense development program has been developed with this company to optimize the extrusion parameters and in particular to minimize (at less than 10\%) the variation of the mechanical properties in the region corresponding to the change of billet. This result has been obtained by heating the block of the die and adding a heatedair oven which keeps constant the temperature of the section, before quenching it, also when the press is stopped for the change of billet. At the beginning of August the sections for the commissioning of the EBW plant and the production of the first conductor length were produced. From January 2001, after the production of the first conductor unit length, the batch production of the remaining 40 sections will be launched.

- The production of the Electron Beam Welding (EBW) plant, as well as the production of the 21 lengths of finished conductor has been awarded by ETH-Z to Techmeta, MetzTessy (France). This company is a leading company in the field of EBW equipment and has specific experience in the fabrication of components that need continuous EBW operations.

The construction of most of the components of the EBW line is well advanced, as shown in Fig. 2, and the commissioning of the plant is foreseen before the end of 2000. The components of the conductor will be delivered on standard spools, which will be installed in the EBW plant, and, after straightening and cleaning, they will be welded inside a vacuum chamber equipped with $2 \mathrm{~EB}$ guns.

After welding the conductor will be machined on the four sides to obtain the right dimension and tolerances. The quality of the welding will be monitored by TV-cameras, and, at after machining an US inspection will be done and the result will be checked and recorded. The conductor will be produced in unit lengths of $2650 \mathrm{~m}$.

\section{WINDING}

The Cold mass will be produced in five modules that will be assembled at CERN after a preliminary coupling at the winding company premises. The winding operation is under the technical and financial responsibility of INFN-Genova with the participation and coordination of CERN and CEASaclay. The contract for the winding operation has been awarded to ANSALDO, Genova (Italy), after an international tender managed by INFN. The specification for the tender was based on the experience gained during the last two years from a large pre-industrialization program aimed at investigating the potential problem in the bending process of a conductor as stiff as the one used for CMS.

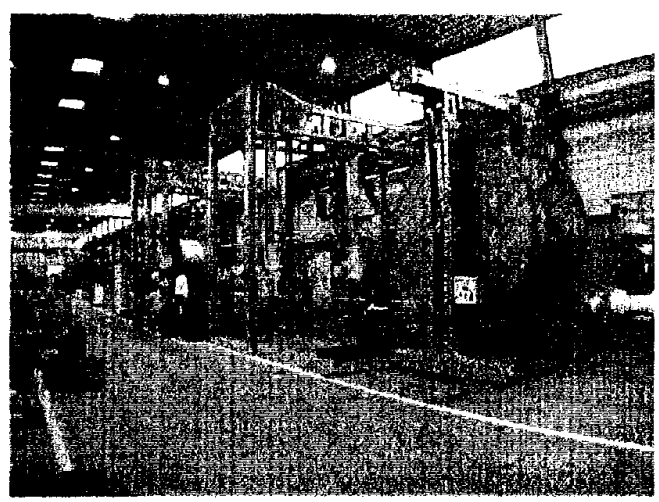

Fig. 2. Construction of the Continuous Electron Beam Welding Plant for the CMS conductor reinforcement. 
The pre-industrialization program has evaluated different alternatives to get the wound pack and investigated aspects related to the keystoning of the conductor, the insulation and the impregnation of the cold mass. For the preindustrialization, the whole unit length of conductor has been bent in a separate operation before transferring it into the mandrel [4].

In the final process a continuous bending and transferring line will be adopted. A sketch of the proposed machine is given in Fig. 3.

The contract for the winding foresees the production of a $0.7 \mathrm{~m}$ high model, full diameter, which will permit to check in detail, and with the final tooling, all the aspects of the fabrication process.

The schedule foresees the commissioning of the winding machine in the first half of 2001 and the completion of the model coil in autumn 2001. The production of the five modules will be completed towards mid 2003. Each module will be coupled with the following one at ANSALDO premises before being transported to CERN where the module to module junctions will be executed. The winding contract is monitored by an ad-hoc group, under the leadership of INFN with the participation of CERN and CEA, which reports regularly to the CMS Magnet Project Management.

\section{ENGINEERING ACTIVITIES AT SACLAY}

The Saclay team is in charge of the general engineering as well as of the follow up of the remaining contracts needed for the completion of the cold mass. Since 1999 a specific group for the assembly operation of the cold mass has been set up. This working group managed by CEA-Saclay with the participation of CERN and the other institutes reports also regularly to the CMS Magnet Board.

The assembly scenario foresees the assembly of the five modules of the cold mass on a mechanical platform on which the final connection of the conductor and the cryogenic piping will be done. The platform is shown in Fig. 4. The outer thermal screen and insulation will be placed before swiveling the coil and enter it, in a cantilevered way, inside the outer shell of the vacuum tank. The specific tooling for the swiveling operation has been specified by CEA-Saclay and is being tendered by KODEL, the HEP institute of Korea.

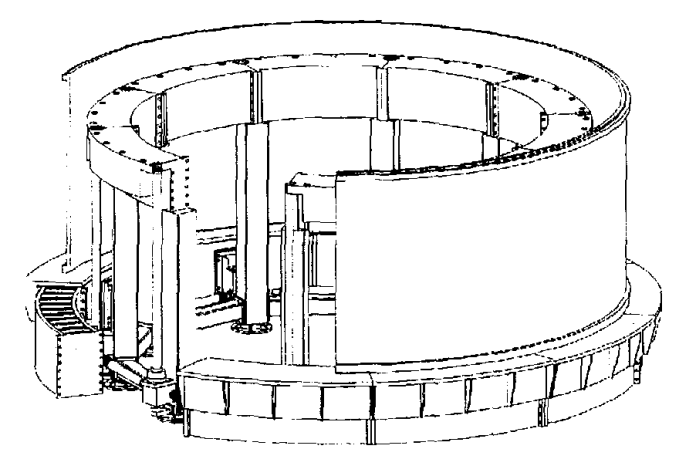

Fig. 3. Final winding machine for the production of the 5 modules of the CMS cold mass.

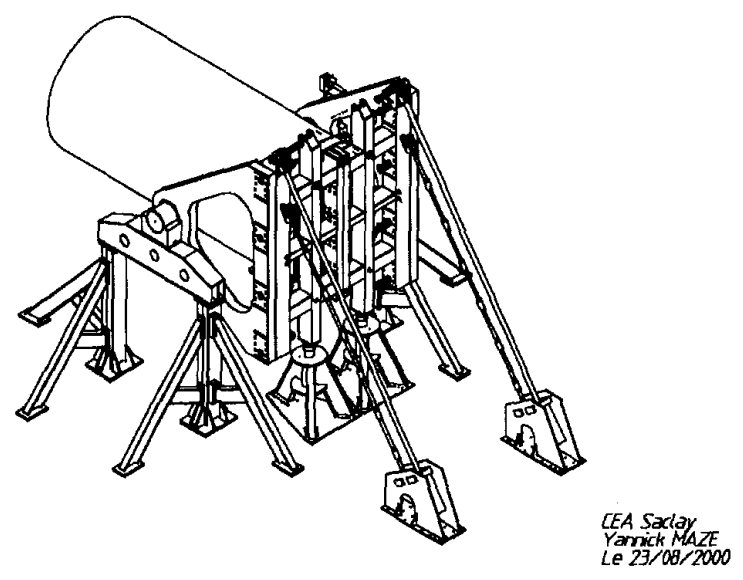

Fig. 4. Swiveling tool for the Cold Mass insertion into the Vactank.

In Saclay during the last year other important subjects have been addressed, in particular:

- The fast discharge voltage has been lowered to $600 \mathrm{~V}$, reducing the value of the dump resistor from 50 to $30 \mathrm{~m} \Omega$, but still keeping the nominal design voltage level for the insulation to $1 \mathrm{kV}$.

- Different tests have been performed to choose the process for the surface preparation of the conductor before insulation and impregnation. The anodic oxidation process could have been the optimum but the sandblasting technique is more easily managed by industry.

- Optimization in the choice of the material for the external mandrels of the coil which is now made from AA 5083 in an H 321 state, and flanges obtained by hot roll milling.

- Development and realization of a suspension system mock-up to understand different constructive and mounting parameters. In addition sets of medium size samples of the vertical and longitudinal tie rods, in TA $5 \mathrm{E}$ eli and TA6 V eli, have been made and will be tested at $4.2 \mathrm{~K}$ in a dedicated test facility. It is foreseen to check the mechanical behavior at $4.2 \mathrm{~K}$ of each tie rod of the final production.

- The final design of the double circuit of the inner cryogenic circuit has been finalized, and a mock up is being done to check the operating process to make welding in the rather crowded region of the cryogenic circuit chimney.

- A specific development program and a test station to choose the most appropriate thermal shielding technique have been launched.

- Realization of a test station to test samples of the external junctions of the conductor for the connection of the layers and the modules of the cold mass.

- Definition of the vacuum circuit components and implantation, inside the allowed space attributed to the coil, by the CMS integration team.

- Design of the current leads which can work damageless at full current for 7 ' without cooling.

- Definition of the switch breakers for the powering and discharging circuit.

- Finalization of the internal instrumentation of the cold mass and final scheme of the quench-detection system. 


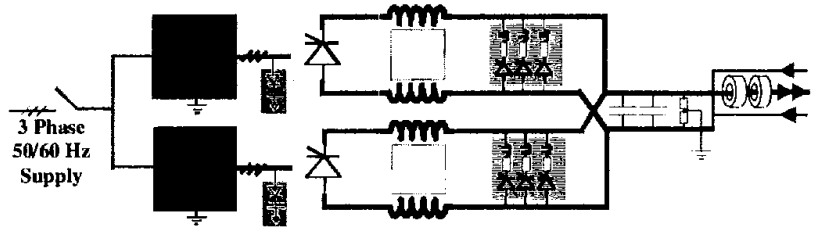

Fig. 5. Scheme of the power supply for the CMS Magnet.

Many of the mentioned activities will continue into the next months, in particular the definition of the final matrix of all the operating conditions of the magnet which will drive the MCS and MSS control scheme (Magnet Control System and Magnet Safety System respectively).

\section{ANCILLARIES}

As mentioned in the introduction some activities are directly managed and executed by CERN with the clear goal of harmonizing the exploitation conditions of the 4 LHC experiments in the context of the LHC machine.

- The contract for the external cryogenic plant has been attributed by CERN to Air Liquide, Sassenage (France) [6]. This contract foresees the delivery of the plant in late 2002, to allow commissioning in the first half of 2003 and be ready for the magnet surface test in autumn 2003.

- The power converter for the feeding circuit has been chosen to be bipolar at $+26 \mathrm{~V},-24 \mathrm{~V}$. Fig. 5 shows the scheme. This choice will permit to drive the magnet also in the discharging phase and use the slow or fast discharge scheme only in case of problems with the power converter itself (or its own auxiliaries like cooling) or the mains. In this way, the time lost for physics in case of any general problem will be minimized, because the field can be lowered and kept to the level corresponding to an acceptable stray field in the zone of the intervention.

- The feeding busbars will be made of copper and made from four water-cooled tubes for each branch. In case of cooling problem the configuration permits to run for more than eight hours with an increase of about $70 \mathrm{C}$ on the copper temperature. The discharging busbars will be made from massive aluminum and positioned in the so-called PM54 shaft to connect the outlet of the cold mass, which will be placed $90 \mathrm{~m}$ underground inside the UX5, and the discharge resistor bank on the surface. The discharge resistors are placed on the surface, as shown in Fig. 6, to avoid large heat dissipations underground which could perturb the ventilation scheme of the LHC tunnel and of the experimental complex.

\section{SCHEDULE AND CONCLUSION}

Most of the Coil Project activities have been achieved as foreseen in the initial planning. Some others, especially those related to large contract tendering or final industrial engagements, have brought a delay of about 5 months in the first part of the Magnet Project. This delay implies that the surface test of the finished magnet will begin toward end of 2003 instead of mid 2003.

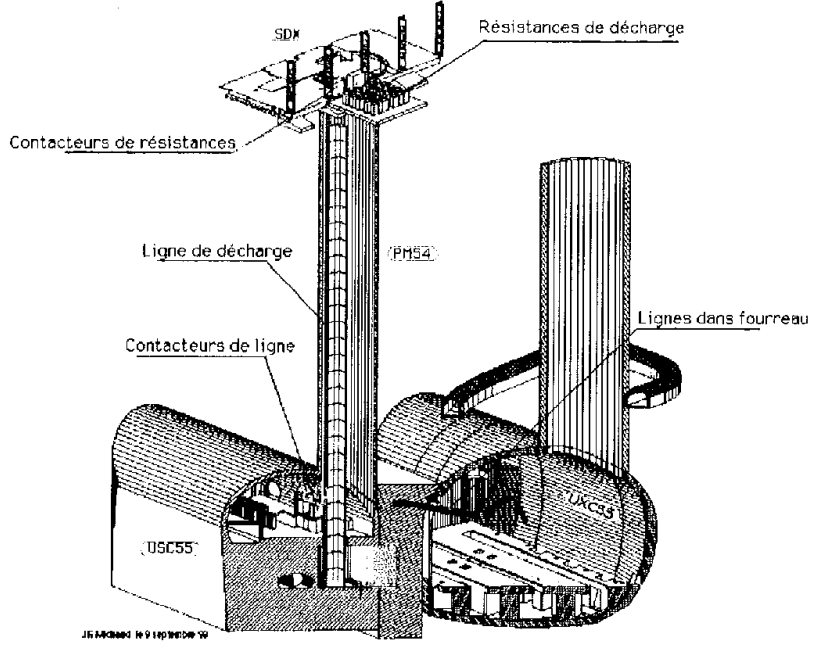

Fig. 6. Ancillaries Layout in the service cavern of the CMS underground experimental complex.

The lowering of the magnet components, which will start before mid 2004 will permit the beginning of the exploitation of a running CMS Detector in September 2005.

The Magnet Project is in good shape financially, and this shoud allow strengthening the assembly team and the planning of additional contracts to introduce some flexibility into the schedule.

\section{REFERENCES}

[1] The CMS Design Team, "The CMS Magnet Technical Design Report", CERN/LHCC report 97-10 (1997).

[2] A. Hervé, "The CMS Detector Magnet," IEEE Trans. on Appl.Supercon Vol.10, No1. pp. 389-394, March 2000.

[3] F. Kircher, P. Bredy, A. Calvo, B. Curé, D. Campi, A. Desirelli, P. Fabbricatore, S. Farinon, A. Hervé, I.L. Horvath, V. Klioukhine, B. Levesy, M. Losasso, J.P. Lottin, R. Musenich, Y. Pabot, A. Payn, C. Pes, C. Priano, F. Rondeaux, S. Sgobba, "Final Design of the CMS Solenoid Cold Mass" IEEE Trans. on Appl.Supercon Vol.10, No1. pp. 407-410 March 2000.

[4] P. Fabbricatore, S. Farinon, R. Musenich, C. Priano, A. Calvo, B. Levesy, F. Rondeaux, M. Perrella, C. D'Urzo, "Pre-Industrialization Activities related to the CMS Coil Winding", this Conference.

[5] I. L. Horvath, B. Dardel, H.P. Marti, J. Neueschwander, R.P. Smith, P. Fabbricatore, R. Musenich, A. Calvo, D. Campi, B. Curé, A. Desirelli, G. Favre, P.L. Riboni, S. Sgobba, T. Tardy, S. Sequeira Lopes Tavares, "The CMS Conductor", IEEE Trans. on Appl. Supercon, Vol 10, No 1 pp. 395-398, March 2000.

[6] D. Delikaris, J.P. Dauvergne, G. Passardi, J.C. Lottin, J.P. Lottin, C. Lyraud, "The Cryogenic System for the Superconducting Solenoid Magnet of the CMS Experiment", Proceeding of MT-15 Conference, Beijing, China - October 1997, Science Press. 